The Be Phenomenon in Early-Type Stars, IAU Colloquium 175

ASP Conference Series, Vol. 214, 2000

M. A. Smith, H. F. Henrichs, and J. Fabregat, eds.

\title{
Doppler Imaging of Non-radially Pulsating Stars
}

\author{
S. Jankov \\ Astronomical Observatory Beograd, Volgina 7, 11050 Beograd, \\ Yugoslavia \\ E. Janot-Pacheco and N.V. Leister \\ Instituto Astronômico e Geofísico, Universidade de São Paulo, \\ C.P. 9638, 01065-970, São Paulo, SP, Brasil
}

\begin{abstract}
In addition to the common applications (temperature, magnetic field, abundance distributions) the Doppler Imaging technique can also be applied to non-radial stellar pulsations (NRP). Due to their rapid rotation, the Be stars are ideal candidates for Doppler Imaging. However, for the stars for which the periodic temporal line profile variability is produced by multiple mode NRP, the classical Doppler image in not an appropriate representation. When the corotating frequencies of existing pulsation modes are non-zero, the reference frame in which the resulting NRP is quasi-stationary cannot be defined. The technique can be applied "mode-by-mode", which is illustrated in time-wavelength and Fourier frequency domain, using the time-resolved sequence of high resolution, high signal-to-noise ratio spectra of the Be star $\zeta$ Oph.
\end{abstract}

\section{Introduction}

The technique of indirect stellar imaging from spectroscopy (Doppler Imaging) allows to obtain, from the line profile disturbances (observed with high signalto-noise ratio, spectral resolution and adequate phase coverage), information about spatial distribution of quasi-stationary structures in stellar atmospheres and environments. This approach is based on the fact that the apparent disk of a rotating star is spatially resolved in the direction of the dispersion axis of the spectrograph, and a surface element defined by the stellar azimuth angle $\phi$ and colatitude $\theta$ is mapped onto a wavelength position corresponding to its Doppler shift: $V_{r}=\left(V_{\mathrm{e}} \sin i\right) \sin \theta \sin \phi$, where $V_{\mathrm{e}} \sin i$ is the projected rotational velocity. The Doppler Imaging techniques have been developed for imaging of local brightness, local abundances and magnetic fields (eg. Rice 1996), but not for imaging of velocity fields. The classical Doppler Imaging was applied to non-radial stellar pulsations in the special case of mono-mode pulsation and dominance of temperature variations (Berdyugina et al. 1999).

The non-radial pulsator model describes the time $(t)$ dependent surface brightness and velocity field distribution $D$ in terms of the pulsation mode fre- 

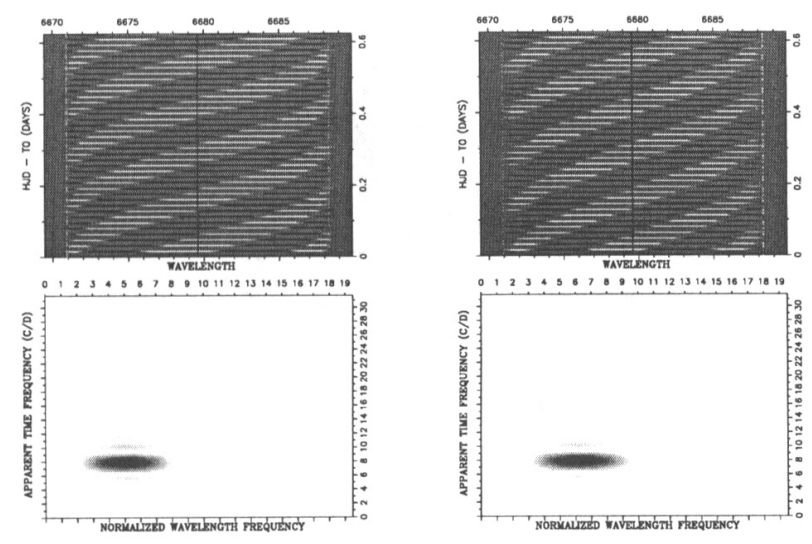

Figure 1. Top: The residual spectra for equatorialy confined $|m|=5$, $l=5$ model (left) and $|m|=5, l=6$ model (right). Bottom: The corresponding Fourier-Doppler images.

quency and quantum numbers:

$$
D=f\left(\phi, \theta, k_{n}, m, l\right) e^{i(\omega t+m \phi)+\Phi_{\mathrm{c}}},
$$

where the ratio of horizontal to vertical velocity $k_{n}$ (depending on the radial order $n$ ), degree $l$, azimuthal order $m$, the apparent time frequency $\omega$ and phase $\Phi_{c}$ define a single pulsation mode and $i$ is the imaginary unity. Strictly speaking, Doppler Imaging of non-radial pulsations requires a new technique of imaging the velocity fields but in a very rapidly rotating star, the pulsation velocity or (and) temperature field(s) act as a small perturbation of the dominant rotation velocity field and the concept of one-to-one mapping holds in this approximation.

\section{Fourier-Doppler Imaging}

The form of Eq. 1 leads to the two-dimensional Fourier analysis in both time and space where the wavelength is mapped onto stellar longitude $\phi$ using: $\phi_{r}=\sin ^{-1}\left\{V_{r} /\left(V_{\mathrm{e}} \sin i \sin \theta\right)\right\}$ where $V_{r}$ is the velocity with respect to the line center, corresponding to the wavelength bin $r$. Assuming that the oscillations are confined to the equatorial region $(\sin \theta \approx 1)$, which is particularly true for sectoral $|m|=l$ oscillations, the normalized wavelength frequency can be associated with the azimuthal order of the pulsation modes. However, it was found experimentally that in the general case, when tesseral modes $|m|<l$ are present, the normalized wavelength frequency more closely represents the nonradial degree $l$ rather than the azimuthal order $m$ (Kennelly et al. 1996). This is illustrated in Fig. 1 where the synthetic residual spectra (mean spectrum subtracted from individual spectra) were produced using the non-radial pulsation models presented in Fig. 2 (top). 

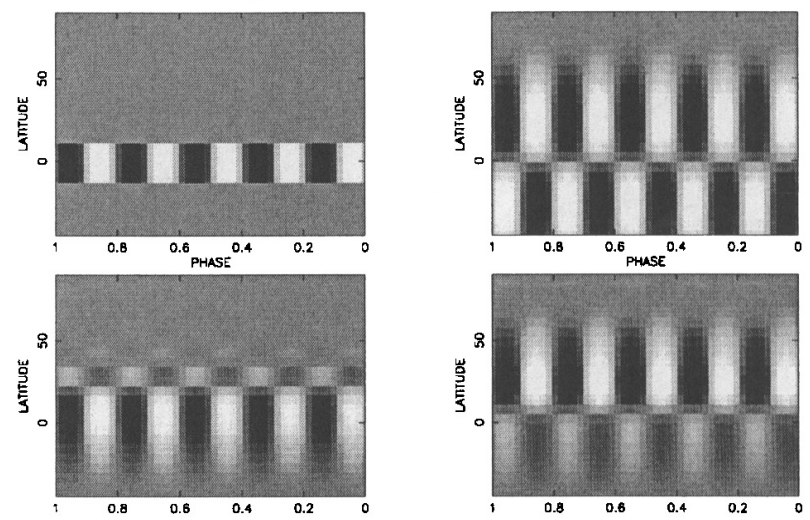

Figure 2. Top: The input images representing the NRP models $|m|=$ $5, l=5$ (left) and $|m|=5, l=6$ (right) used to produce residual spectra shown in Fig. 1 (top). Bottom: The Doppler Imaging reconstructions from these spectra. For the $|m|=5, l=5$ model the distortions far from the equator are canceled out to simulate the effect of fast rotation (Townsend 1997).

\section{Doppler Image reconstructions of non-radial pulsations}

The main advantage of the Fourier-Doppler Imaging technique is that it decomposes the complex line profile variations in the case of multi-mode pulsation, where the resultant distribution can be represented as a sum of terms described by eq. 1 . But the technique does not produce classical images, so the FourierDoppler images presented in Fig. 1 cannot tell us whether it is the case of the sectoral mode $|m|=l$ or tesseral mode $|m|<l$. Fig. 2 presents the input images and Doppler Imaging reconstructions using the same spectra as presented in Fig. 1 (top). From this reconstruction the different modes can be clearly distinguished.

However, the Doppler Imaging approach cannot be applied directly to the line profile variations produced by the multi-mode pulsations. The apparent time frequency $\omega$ is related to the corotating frequency $\omega_{\mathrm{c}}$ and the rotational frequency of the star $\Omega_{\mathrm{r}}$ by the relation (Ledoux 1951): $\omega=\omega_{\mathrm{c}}-m \Omega_{\mathrm{r}}$. Introducing $g=\left(\omega_{\mathrm{c}} / \omega-1\right)^{-1}$ this equation can be rewritten as: $\omega=-m g \Omega_{\mathrm{r}}=-m \Omega_{\mathrm{w}}$, where $\Omega_{\mathrm{w}}=g \Omega_{\mathrm{r}}$ is the rotational frequency of the wave. The Doppler Imaging technique requires the reference frame related to the rotational frequency of the structure to be imaged. In the reference frame of one wave another wave is not stationary, so the basic condition for Doppler Imaging is not fulfilled.

\section{Application to multiple mode NRP}

Fortunately, the non-radial pulsator model describes the surface intensity distribution and velocity field in terms of a few parameters; the "image" associated with a single mode can in practice be described by the pulsation degree and az- 

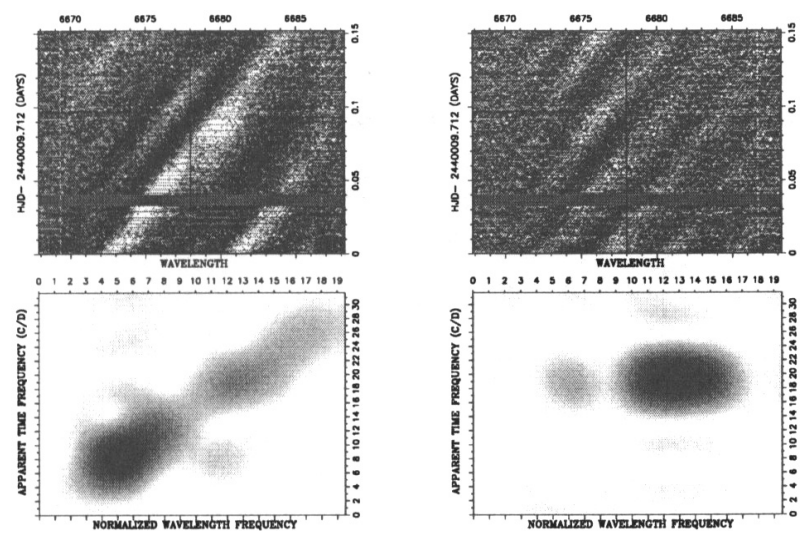

Figure 3. Top: The residual spectra of $\zeta$ Oph (left) whitened with apparent time frequencies 7.2, 11.9 and $26 \mathrm{c} / \mathrm{d}$ (right). Bottom: The corresponding Fourier-Doppler images.

imuthal order and extracted at the appropriate time frequency. We illustrate the corresponding procedure using data of $\zeta$ Oph obtained at Laboratório Nacional de Astrofísica, Brazil. Fig. 3 shows the irregular residual spectra (top-left) revealing the complex underlying structure. The resulting Fourier-Doppler image (bottom-left) shows clearly the multi-mode nature of the line profile variations. The low frequency modes can be related to the pulsations with apparent time frequencies 7.2, 11.9 and 19.2 cycles per day (c/d), determined by Kambe et al. (1997). Subtracting these frequencies from the data, in each wavelength bin separately, we obtain the very regular residual spectra. Only such monomode residual spectra can be used to obtain a Doppler image of the pulsation. The corresponding Fourier-Doppler image shows a single mode with apparent time frequency $26 \mathrm{c} / \mathrm{d}$. Subtracting $7.2,11.9$ and $26 \mathrm{c} / \mathrm{d}$ frequencies from original residual spectra one obtains the whitened residual spectra shown in Fig. 3 (top-right), which can be used for Doppler Imaging of the $19.2 \mathrm{c} / \mathrm{d}$ pulsation. Similarly, the data can be processed for Doppler Imaging of the pulsations characterized by different apparent time frequencies.

\section{References}

Berdyugina, S.V., Korhonen, H., Schrijvers, C., Telting, J.H. 2000, these proceedings

Kambe, E., Hirata, R., Ando, H. et al., 1997, ApJ 481, 406

Kennelly, E.J., Walker, G.A.H., Catala, C. et al., 1996, A\&A 313, 571

Ledoux, P., 1951, ApJ 114, 373

Rice, J.B. 1996, in K.G. Strassmeier and J.L. Linsky, eds., Proc. IAU Symp. 176, Kluwer Academic Publishers, Dordrecht, p. 19

Townsend, R.H.D. 1997, MNRAS 284, 839, 496 